\title{
INFLUÊNCIA DA ALTITUDE NA DEFORMAÇÃO DE ÁREAS PROJETADAS NOS SISTEMAS PLANOS UTM, LTM E RTM
}

\section{INFLUENCE OF ALTITUDE IN DEFORMATION OF PROJECTED AREAS IN UTM, LTM AND RTM PLANS SYSTEMS}

\begin{abstract}
Leonard Niero da Silveira ${ }^{1}$ Jonatan dos Santos Rocha ${ }^{2}$

\section{Resumo}

Atualmente, o sistema de projeção cartográfica mais utilizado é o UTM, por propor o uso de fórmulas simplificadas para cálculo que podem ser usadas facilmente em campo além de proporcionar precisões suficientes para a maioria dos trabalhos, com ressalvas quanto à utilização em obras de engenharia. Para obras que necessitem de uma melhor precisão para locação, eliminando as distorções inerentes a transformação de elementos sobre a superfície elipsóidica para a superfície plana, a melhor opção é a conversão das coordenadas planas UTM em coordenadas plano topográficas locais (levando em consideração a altitude), sendo admitidos em alguns tipos de serviços também os sistemas de projeção LTM e RTM. Na comparação feita, ao nível do mar a área que mais se aproximou do valor calculado para plano Topográfico Local foi o sistema LTM seguido pelo RTM e a maior diferença para o UTM. A comparação feita em altitude a que mais se aproximou da área computada do plano Topográfico Local foi a RTM, seguido pela LTM e a maior diferença permaneceu sendo a UTM. Analisando-se as diferenças relativas observou-se que houve uma ligeira piora nos resultados levando-se em consideração a altitude.
\end{abstract}

Palavras-chave: Topografia. Geodésia. Cartografia. Sistemas de Projeção.

\begin{abstract}
Currently, the cartographic projection system most used is the UTM, by proposing the use of simplified equations for calculation that can be easily used in the field as well as providing sufficient explanation for most of the work, with restrictions on the use of engineering works. For works that require better accuracy for location, eliminating the distortions inherent in the transformation of elements from a ellipsoidal surface to the plan surface, the best option is a conversion of the plan coordinates UTM in topographical local plan coordinates (taking into account the altitude), being accepted in some types of works also LTM and RTM projection systems. In comparison made, at sea level the area closest to the calculated value for Topographical Location plan was the LTM system followed by the RTM and the biggest difference for UTM. The comparison made in altitude that most came from the Topographical Location plan computed area was the RTM, followed by the LTM and the biggest difference remained the UTM. Analyzing the relative differences observed that there was a slight worsening of the results taking into account the altitude.
\end{abstract}

Keywords: Topography. Geodesy. Cartography. Projection Systems.

\footnotetext{
${ }^{1}$ Engenheiro Agrimensor, Mestre em Geomática, Professor da Universidade do Extremo Sul Catarinense - Unesc; E-mail leonard@amiranet.com.br.

${ }^{2}$ Engenheiro Agrimensor, TOPOGEO Engenharia e Topografia Ltda; E-mail jrochaagri@hotmail.com.
} 


\section{Introdução}

Devido a diversos problemas inerentes à aplicação dos sistemas de projeção cartográfica em trabalhos de implantação de obras civis, efetuou-se a comparação entre os sistemas UTM, RTM, LTM e Topográfico Local, em um levantamento de uma área ao nível do mar, a aproximadamente 43 metros de altitude, e um em uma área a 1.425 metros de altitude, verificando a diferença e precisão de cada uma das situações considerando o plano topográfico local como referência, sendo feita uma avaliação de qual sistema se torna mais adequada dependendo da situação.

Discutir as possibilidades metodológicas, suas vantagens e deficiências, para a transformação de coordenadas de uma projeção cartográficas em topográficas é de suma importância para facilitar o seu emprego e o aperfeiçoamento dessas transformações, de modo que, a exatidão seja compatível com as especificações estabelecidas para cada trabalho.

Por isso é necessária à quantificação destas distorções em diferentes níveis (altitudes) para que sirva de alerta para os profissionais que efetuam os cálculos de área com coordenadas em projeções cartográficas sem efetuar a redução destas coordenadas para o plano topográfico local, ou seja, transformar distâncias planas inerentes às projeções cartográficas para distâncias no plano horizontal local.

\section{Fundamentação teórica}

\subsection{Sistema de projeção plana Topográfica Local}

Segundo Tuler e Saraiva (2014 apud ABNT, 1994), é o sistema de projeção utilizado nos levantamentos topográficos pelo método clássico, para a representação das posições relativas dos elementos levantados por meio de medições angulares e lineares, horizontais e verticais, cujas características são:

- As projetantes são ortogonais à superfície de projeção, significando estar o centro de projeção localizado no infinito;

- A superfície de projeção é um plano normal à vertical do lugar no ponto da superfície terrestre considerado como origem do levantamento, sendo seu referencial altimétrico referido ao datum vertical brasileiro de Imbituba/SC; 
- As deformações máximas inerentes à desconsideração da curvatura terrestre e à refração atmosférica têm as seguintes expressões aproximadas:

$$
\begin{array}{ll}
\circ & \Delta \mathrm{l}(\mathrm{mm})=-0,004 \mathrm{l}^{3}(\mathrm{~km}) ; \\
\circ & \Delta \mathrm{h}(\mathrm{mm})=+78,5 \mathrm{l}^{2}(\mathrm{~km}) ; \\
\circ & \Delta \mathrm{h}^{\prime}(\mathrm{mm})=+67 \cdot \mathrm{l}^{2}(\mathrm{~km}) .
\end{array}
$$

Onde:

$\Delta \mathrm{l}=$ deformação planimétrica devida à curvatura da Terra, em mm;

$\Delta \mathrm{h}=$ deformação altimétrica devida à curvatura da Terra, em mm;

$\Delta \mathrm{h}^{\prime}=$ deformação altimétrica devida ao efeito conjunto da curvatura da Terra e da refração atmosférica, em mm;

1 = distância considerada no terreno, em km;

- O plano de projeção tem a sua dimensão máxima limitada a $50 \mathrm{~km}$, a partir da origem (ABNT, 1998), de maneira que o erro relativo, decorrente da desconsideração da curvatura terrestre, não ultrapasse 1/35000 nesta dimensão e 1/15000 nas imediações da extremidade desta dimensão. Deve-se estabelecer subdivisão de glebas do sistema, quando o desnível for superior a $150 \mathrm{~m}$ para mais ou para menos na área a ser representada;

- A localização planimétrica dos pontos, medidos no terreno e projetados no plano de projeção, se dá por intermédio de um sistema de coordenadas cartesianas, cuja origem coincide com a do levantamento topográfico;

- O eixo das ordenadas é a referência azimutal que, dependendo das peculiaridades do levantamento, pode estar orientado para o norte geográfico, para o norte magnético ou para uma direção notável do terreno, julgada importante.

\subsection{Sistemas de projeção cartográfica}

$\mathrm{Na}$ impossibilidade de se desenvolver uma superfície esférica ou elipsóidica sobre um plano sem deformações, buscam-se soluções para a representação de uma área 
em uma superfície curva num plano, portanto utilizam-se projeções tais que permitam diminuir ou eliminar parte das deformações conforme a aplicação desejada.

Segundo Duarte (1995), a projeção cartográfica é a base para a construção de mapas. No entanto, as projeções também podem ser utilizadas para a confecção de produtos cartográficos de maior escala (plantas e cartas).

Estas projeções nada mais são do que o resultado de um conjunto de operações que permite colocar no plano, fenômenos inscritos numa esfera ou, no caso da Terra, representado por um elipsoide que é a figura matemática que imita a verdadeira forma da terra.

Figura 1: Projeção da superfície terrestre até o sistema de coordenadas plano.

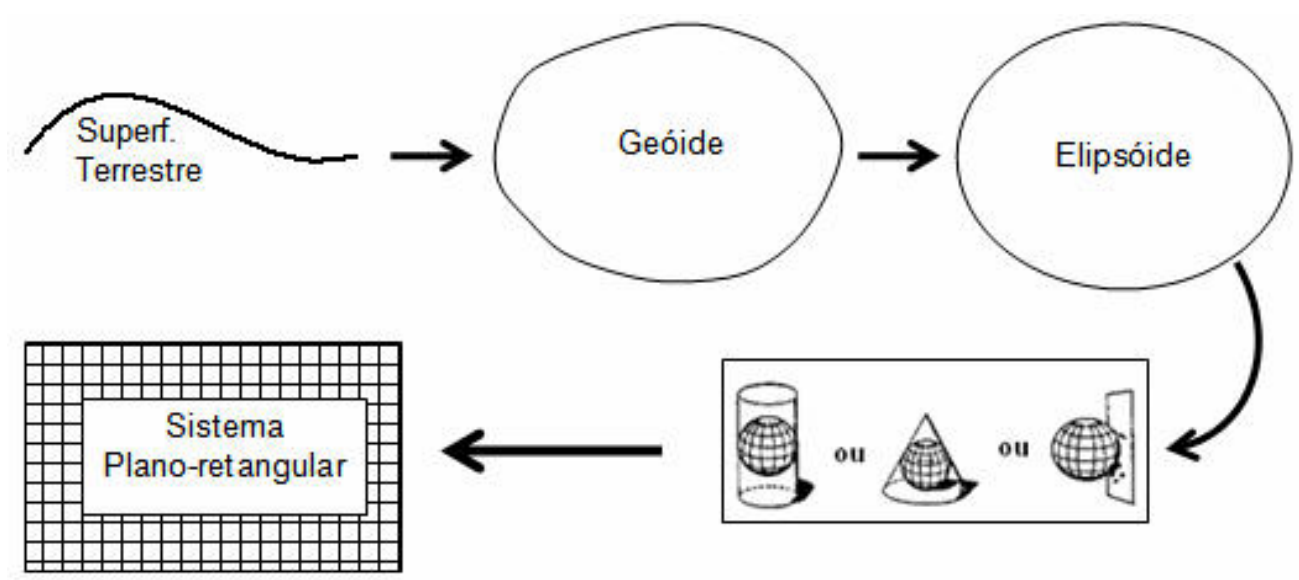

Fonte: França (2006, p.6).

Transformação das coordenadas planas UTM em coordenadas no Plano Topográfico, por meio do fator de escala $\left(K_{R}\right)$.

- Distância UTM entra ponto e Base $\left(D_{u t m}\right)$

$D_{U T M}=\sqrt{E_{i}-E_{B}^{z}+N_{i}-N_{B}^{z}}$

-Cálculo do azimute plano UTM (Az' $\left.{ }_{\text {UTM }}\right)$

$\operatorname{Cos} A z_{\text {UTM }}^{\prime}=\left(N_{i}-N_{B}\right) \div D_{U T M}$

Se $\Delta \mathrm{E}<0, \mathrm{Az}_{\mathrm{UTM}}=360^{\circ}-\mathrm{Az}^{\prime}{ }_{\mathrm{UTM}}$, se $\Delta \mathrm{E}>0 \mathrm{Az}_{\mathrm{UTM}}=\mathrm{Az}^{\prime}{ }_{\mathrm{UTM}}$

- Cálculo do azimute topográfico $\left(\mathrm{Az}_{\mathrm{top}}\right)$ 
$A z_{\text {TOP }}=A z_{\text {UTM }} \pm \angle$ rotaçâo

Se $A z_{\text {top }}<0, A z_{\text {top }}+360^{\circ}$, se $A z_{\text {top }}>360, A z_{\text {top }}-360^{\circ}$

- Cálculo da latitude média $\left(\phi_{\mathrm{m}}\right)$

$\phi_{\mathrm{m}}=\left(\phi_{\mathrm{i}}+\phi_{\mathrm{B}}\right) \div 2$

- Cálculo da longitude média $\left(\lambda_{m}\right)$

$\lambda_{\mathrm{m}}=\left(\lambda_{\mathrm{i}}+\lambda_{\mathrm{B}}\right) \div 2$

- Fator de escala médio $\left(k_{m}\right)$

$\mathrm{K}_{\mathrm{m}}=\mathrm{K}_{0} \div\left(\sqrt{1-\left(\operatorname{Cos} \phi_{\mathrm{m}} \times \operatorname{Sen}\left(\lambda_{\mathrm{m}} \times \lambda_{\mathrm{mc}}\right)\right.}\right)^{2}$

- Cálculo da primeira excentricidade ( $\left.\mathrm{e}^{2}\right)$

$e^{2}=\left(a^{2}-b^{2}\right) \div a^{2}$

- Cálculo do Raio Médio $\left(\mathbf{R}_{\mathrm{m}}\right)$

$R_{m}=a \times \sqrt{\left(1-e^{2}\right)} \div\left(1-e^{2} \times \operatorname{Sen}^{2} \phi_{m}\right)$

- Cálculo do Fator de Escala $\left(K_{R}\right)$

$\mathrm{K}_{\mathrm{r}}=\mathrm{R}_{\mathrm{m}} \div\left(\mathrm{R}_{\mathrm{m}}+\mathrm{H}_{\mathrm{m}}\right)$

- Distância topográfica $\left(D_{\text {top }}\right)$

$\mathrm{D}_{\mathrm{TOP}}=\mathrm{D}_{\mathrm{UTM}} \div \mathrm{Kr}$

- Cálculo das coordenadas Topográficas $\left(\mathbf{X}_{\mathbf{i}} ; \mathbf{Y}_{\mathbf{i}}\right)$

$X_{i}=\left(\operatorname{Sen} A z_{T O P} \times D_{T O P}\right)+X_{B}$
$Y_{i}=\left(\operatorname{Cos} A z_{T O P} \times D_{T O P}\right)+Y_{B}$

Onde:

$\mathrm{D}_{\mathrm{UTM}}$ : Distância plana UTM entra o ponto e a base;

$\mathrm{Az}_{\mathrm{UTM}}$ : Cálculo do azimute plano UTM;

$\mathrm{Az} z_{\text {top }}$ : Cálculo do azimute topográfico;

$\phi_{\mathrm{m}}$ : latitude média;

$\lambda_{\mathrm{m}}$ : longitude média;

$\mathrm{k}_{\mathrm{m}}$ : Fator de escala médio;

$\mathrm{e}^{2}$ : Primeira excentricidade; 
$\mathrm{R}_{\mathrm{m}}$ : Raio médio;

$\mathrm{K}_{\mathrm{r}}$ : Fator de Escala;

$\mathrm{D}_{\text {top }}$ : Distância topográfica;

$\mathrm{X}_{\mathrm{i}} ; \mathrm{Y}_{\mathrm{i}}$ : Coordenadas Topográficas;

$\mathrm{E}_{\mathrm{i}} ; \mathrm{E}_{\mathrm{B}} ; \mathrm{N}_{\mathrm{i}} ; \mathrm{N}_{\mathrm{B}}$ : Coordenadas planas UTM.

\section{Material e métodos}

Foram efetuados dois levantamentos com receptores GNSS, sendo um próximo ao nível do mar (com altitude de aproximadamente 43 metros) na cidade de torres- RS e outro em Bom Jardim da Serra- SC (com altitude de aproximadamente 1.425 metros).

No levantamento foram utilizados tripé, bastão e piquetes. Foi efetuado o levantamento das áreas com um receptor GNSS marca Topcon, modelo Hiper Lite Plus com capacidade de rastreio dos satélites dos sistemas GPS e Glonass, com precisão nominal de $3 \mathrm{~mm} \pm 1 \mathrm{ppm}$ da linha de base.

No processamento dos dados foram utilizados os softwares Topcon Tools, o Microsoft Excel rodando o aplicativo de transformação de coordenadas Transfogeo e o Autodesk AutoCad para elaboração dos desenhos.

Foi efetuado o levantamento de todo o perímetro utilizando o método cinemático stop and go com inicialização do receptor móvel de cinco minutos para uma distância do receptor base não superior a cem metros.

Os dados coletados foram descarregados e processados a partir das coordenadas autônomas da base, pois não é necessário referenciá-la ao sistema geodésico oficial, o objetivo é comparar a área a partir de um levantamento local.

Os dados foram processados e transformados no software Topcon Tools em coordenadas no sistema de projeção transversa de mercartor (UTM).

Foram determinadas as áreas em coordenadas planas UTM a partir dos desenhos efetuados. Também foram determinadas as áreas a partir das coordenadas nos sistemas planos LTM e RTM, cujas coordenadas foram transformadas utilizando o aplicativo Transfogeo a partir das coordenadas planas UTM.

Também foram determinadas as coordenadas no plano topográfico local, calculadas a partir das coordenadas planas UTM, convertendo-se as distâncias planas UTM a partir do coeficiente de deformação linear (K) e do fator de altitude (c), gerando dessa forma o fator de escala (Kr). Também foram determinados os azimutes verdadeiros a partir dos azimutes planos e do cálculo da convergência meridiana. 


\section{Análise dos resultados}

Quanto ao levantamento da área ao nível do mar, foi computada a medida agrária (em metros quadrados) obtendo-se o valor de $22.265,2301 \mathrm{~m}^{2}$.

A partir dos vértices em coordenadas planas UTM foi efetuada a conversão para os sistemas planos LTM e RTM além do plano Topográfico Local, que é o valor que traduz com mais fidelidade o verdadeiro valor da área.

Os valores de área calculados para os demais sistemas foram:

- $\quad$ RTM: $22.278,2901 \mathrm{~m}^{2}$

- LTM: $22.275,5394 \mathrm{~m}^{2}$

- Topográfica Local: 22.275,695 $\mathrm{m}^{2}$

Quanto ao levantamento da área no plano em altitude elevada, foi obtido o valor de $11.549,3139 \mathrm{~m}^{2}$ para as coordenadas no sistema plano UTM.

Para os demais sistemas, foram obtidos os seguintes valores:

- RTM: $11.552,2862 \mathrm{~m}^{2}$

- LTM: $11.551,4671 \mathrm{~m}^{2}$

- Topográfica Local: $11.558,0284 \mathrm{~m}^{2}$

A partir desses valores foi comparada a área do plano Topográfico Local com as áreas dos demais sistemas retornando as diferenças apresentadas nos quadros 1 e 2 .

Quadro 1: Análise de dados de Torres- RS

\begin{tabular}{|l|c|c|c|}
\hline \multicolumn{1}{|c|}{ ANÁLISE DE DADOS TORRES- RS } \\
\hline \multicolumn{1}{|c|}{ SISTEMA DE COORDENADAS } & ÁREA TOTAL & UNIDADE & DIFERENÇAS \\
\hline UTM & $22.265,2301$ & $\mathrm{~m}^{2}$ & 10,4649 \\
\hline Comparação Topográfica x UTM ( relativa) & & $\mathrm{m}^{2} / \mathrm{m}^{2}$ & 0,000469790 \\
\hline RTM & $22.278,2901$ & $\mathrm{~m}^{2}$ & 2,5951 \\
\hline Comparação Topográfica $x$ RTM ( relativa) & & $\mathrm{m}^{2} / \mathrm{m}^{2}$ & 0,000116499 \\
\hline LTM & $22.275,5394$ & $\mathrm{~m}^{2}$ & 0,1556 \\
\hline
\end{tabular}




\begin{tabular}{|l|c|c|c|} 
Comparação Topográfica x LTM ( relativa) & & $\mathrm{m}^{2} / \mathrm{m}^{2}$ & 0,000006985 \\
\hline TOPOGRÀFICA LOCAL & $22.275,6950$ & $\mathrm{~m}^{2}$ & \\
\hline
\end{tabular}

Quadro 2: Análise de dados de Bom Jardim da Serra- SC

\begin{tabular}{|l|c|c|c|}
\hline \multicolumn{3}{|c|}{ ANÁLISE DE DADOS BOM JARDIM DA SERRA- SC } \\
\hline \multicolumn{1}{|c|}{ SISTEMA DE COORDENADAS } & ÁREA TOTAL & UNIDADE & DIFERENÇAS \\
\hline UTM & $11.549,3139$ & $\mathrm{~m}^{2}$ & 8,7145 \\
\hline Comparação Topográfica x UTM ( relativa) & & $\mathrm{m}^{2} / \mathrm{m}^{2}$ & 0,000753978 \\
\hline RTM & $11.552,2862$ & $\mathrm{~m}^{2}$ & 5,7422 \\
\hline Comparação Topográfica x RTM ( relativa) & & $\mathrm{m}^{2} / \mathrm{m}^{2}$ & 0,000496815 \\
\hline LTM & $11.551,4671$ & $\mathrm{~m}^{2}$ & 6,5613 \\
\hline Comparação Topográfica x LTM ( relativa) & & $\mathrm{m}^{2} / \mathrm{m}^{2}$ & 0,000567683 \\
\hline TOPOGRÁFICA LOCAL & $11.558,0284$ & $\mathrm{~m}^{2}$ & \\
\hline
\end{tabular}

Como a áreas levantadas não tem valores iguais, foi feito a comparação sobre o valor relativo, ou seja, o valor de erro em metros quadrados por área computada no plano Topográfico Local.

Foi feita também uma avaliação gráfica dos resultados alcançados como mostra as figuras $1 \mathrm{e} 2$.

Figura 1: Avaliação gráfica da área de Torres- RS 


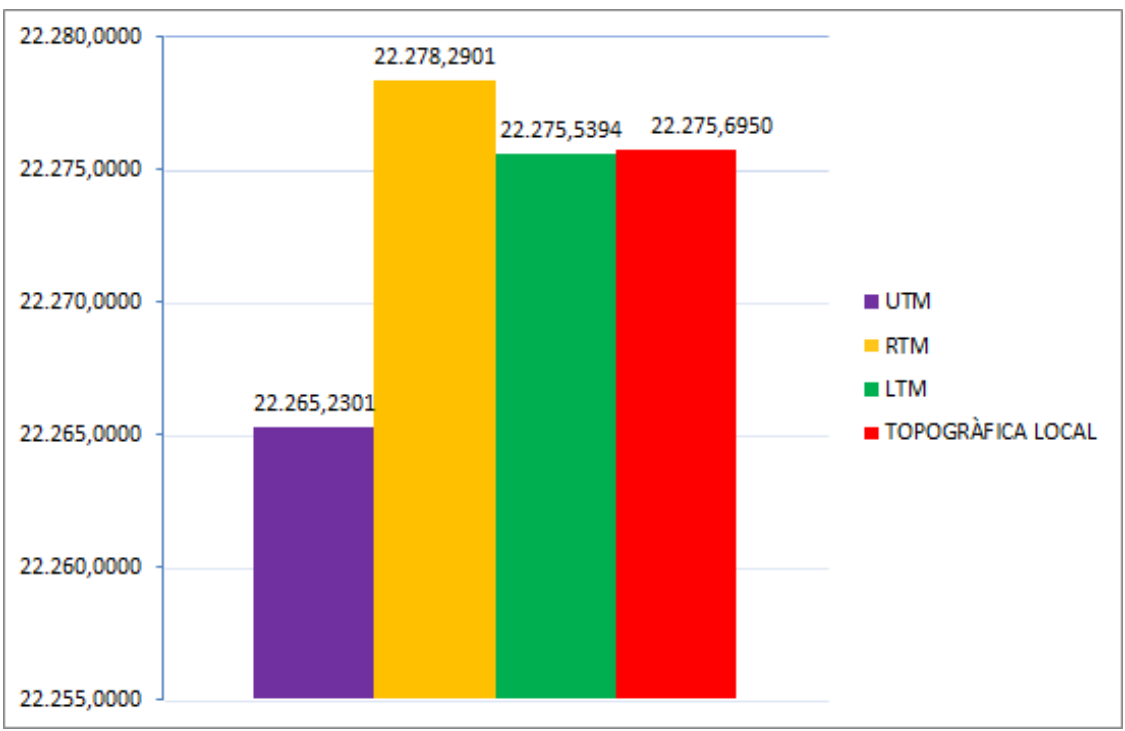

Figura 2: Avaliação gráfica da área de Bom Jardim da Serra- SC

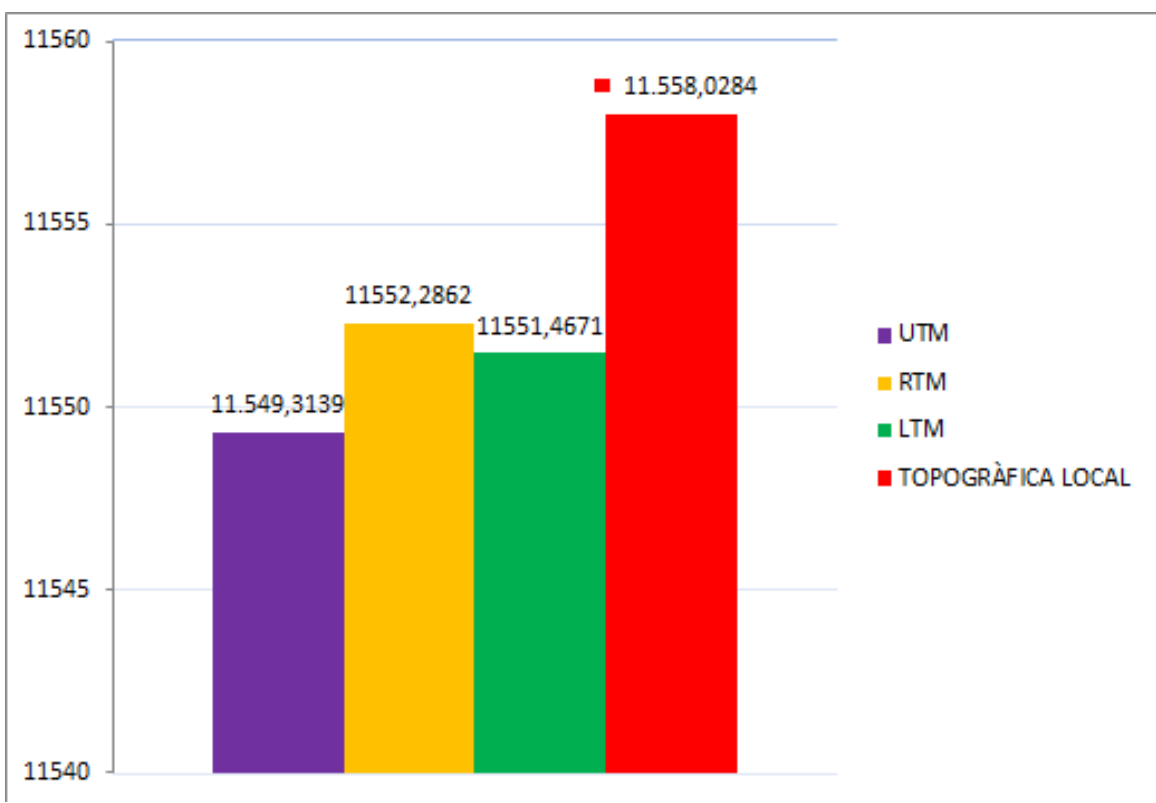

Como é possível observar na comparação feita, para o nível do mar a área que mais se aproximou do valor calculado para plano Topográfico Local foi o sistema LTM seguido pelo RTM e a maior diferença para o UTM.

A comparação feita em altitude a que mais se aproximou da área computada do plano Topográfico Local foi a RTM, seguido pela LTM e a maior diferença permaneceu sendo a UTM.

Analisando-se as diferenças relativas observou-se que houve uma ligeira piora nos resultados levando-se em consideração a altitude, como por exemplo, a diferença relativa entre o sistema LTM x Topográfico Local no nível do mar que ficou 
$0,000006985 \mathrm{~m}^{2} / \mathrm{m}^{2}$, já na área em altitude levando em consideração a mesma comparação o resultado obtido foi de $0,000567683 \mathrm{~m}^{2} / \mathrm{m}^{2}$, no sistema RTM x Topográfico Local no nível do mar obteve-se $0,000116499 \mathrm{~m}^{2} / \mathrm{m}^{2}$, e em altitude foram 0,000496815, ou seja, as diferenças relativas entre os valores de áreas determinadas em Bom Jardim da Serra- SC foram maiores.

\section{Considerações finais}

A escolha de um sistema de projeção não deve ser definida somente pela menor deformação que a mesma apresenta, mas também pela quantidade de área a ser mensurada e pelas exigências do contratante dos serviços.

Observamos que na área ao nível do mar obtiveram-se melhores resultados, devido o fator altitude nesta área, sendo o sistema topográfico local o que mais se aproxima do verdadeiro valor. Estes valores encontrados próximo ao nível do mar são em relação a uma área de $22.275,70 \mathrm{~m}^{2}$ considerando as coordenadas topográficas locais.

Ao nível do mar, as discrepâncias entre os sistemas de projeção UTM, RTM e LTM mostram que em determinadas posições (próximo às bordas do fuso) quanto maior a amplitude, maior será a discrepância em relação ao sistema topográfico local para uma área próxima do nível do mar. No entanto, deve-se considerar que se a área estiver próximo às linhas de secância do cilindro, não importando o tamanho do fuso, a distorção linear é nula e, portanto, os valores projetados nos sistemas UTM, LTM e RTM serão similares aos valores referenciados ao plano topográfico local (ângulos e distâncias). Próximo ao meridiano central, os valores entre os sistemas LTM e RTM não serão diferentes devido ao fator de distorção linear serem iguais $\left(\mathrm{K}_{0}=0,999995\right)$.

Porém, a área em altitude, mostrou que nem sempre a amplitude do fuso é o principal fator que se deve levar em consideração quanto às menores deformações, mas deve-se levar em consideração a posição da área no fuso e sua altitude, os resultados nos mostraram que o sistema RTM obteve melhor resultado quando comparado com o LTM e UTM, sendo o sistema topográfico local o valor mais próximo da verdadeira grandeza. Sendo que o RTM ficou melhor que o LTM porque a posição da área no fuso RTM era mais favorável que a posição no fuso LTM (próximo ao ponto de secância do cilindro onde o coeficiente de deformação linear é nulo). Os valores encontrados para área em 
altitude são em relação a uma área de 11.558,03 $\mathrm{m}^{2}$ considerando as coordenadas topográficas locais.

\section{Referências bibliográficas}

ASSOCIAÇÃO BRASILEIRA DE NORMAS TÉCNICAS. NBR 13133: Execução de levantamento topográfico. Rio de Janeiro, 1994.

ASSOCIAÇÃO BRASILEIRA DE NORMAS TÉCNICAS. NBR 14166: rede de referência cadastral. Rio de Janeiro, 1998.

FRANÇA, Rovane Marcos de. ANÁlISE DE SISTEMAS DE PROJEÇÃO CARTOGRÁFICA EM RODOVIAS DE SANTA CATARINA. 2007. 60 f. Trabalho de Conclusão de Curso (Graduação em Engenharia Civil). Área de atuação Rodovias de Santa Catarina- Universidade do Sul de Santa Catarina, Palhoça.

FRANÇA, Rovane Marcos de. USO DE SISTEMAS DE PROJEÇÃO TRANSVERSA DE MERCATOR EM OBRAS DE ENGENHARIA. V Simpósio Brasileiro de Ciências Geodésicas e Tecnologias da Geoinformação, 2014.

SILVEIRA, Leonard Niero da. Transporte de altitude Ortométrica pelo método Relativo: Geodésia III, Maio. de 2014. Notas de Aula.

SILVEIRA, Luis. Carlos da. Cálculos Geodésicos no Sistema UTM aplicados a Topografia.1ª Ed. Criciúma: Editora Luana, 1990. 